\title{
SOME COEFFICIENT PROBLEMS FOR STARLIKE FUNCTIONS
}

\author{
ALBERT PFLUGER
}

\section{Introduction}

Consider the class $S^{*}$ of normalized starlike functions $f$ in the unit disc $D=\{|z|<1\}$, whose basic results were established by Rolf Nevantinna in his paper [6], and write

$$
f(z)=z+a_{2} z^{2}+\ldots+a_{n} z^{n}+\ldots .
$$

The mapping

$$
A_{n}: f \mapsto\left(a_{2}, \ldots, a_{n}\right)
$$

associates to each $f$ a point in $C^{n-1}$ and takes $S^{*}$ onto some compact set $S_{n}^{*}$ which is called the $n$-th coefficient body for the class $S^{*}$. This paper deals with some basic properties of $S_{n}^{*}$. It will be proved that $S_{n}^{*}$ is homeomorphic to a ball in $C^{n-1}$, that for each boundary point $a$ of $S_{n}^{*}$ there is only one function $f$ in $S^{*}$ such that $A_{n}(f)=a$ and that $A_{n}(f)$ is on the boundary of $S_{n}^{*}$ if and only if $f$ takes the unit disc onto a domain which is bounded by at most $n-1$ rays

$$
R_{j}=\left\{z=t e^{i \alpha_{j}} \mid t \geqq r_{j}\right\}, \quad \alpha_{1}<\alpha_{2}<\cdots<\alpha_{m}<\alpha_{1}+2 \pi,
$$

$1 \leqq m<n$. Contrary to the analogous problem for the class $S$ (cf. [9] for example) the situation here is very explicit and elementary. The basic idea is to consider an analogous coefficient problem for the Carathéodory class $C$ of functions $g$ holomorphic in the unit disc which have positive real part and are normalized by the condition $g(0)=1$. For each $n$ the expansion

$$
g(z)=1+2 c_{1} z+\ldots+2 c_{n} z^{n}+\ldots
$$

defines a mapping

$$
\gamma_{n}: g \mapsto\left(c_{1}, \ldots, c_{n}\right)
$$


of $C$ onto some compact set $C_{n}$ in $C^{n}$, which is called the $n$-th coefficient body for the class $C$. The basic properties of $C_{n}$ (Theorem A) are due to C. Carathéodory and $\mathrm{O}$. Toeplitz; for completeness a full proof will be given in the third part of the paper.

$C_{n}$ is a convex body in $C^{n}$, hence $C_{n}$ admits for each boundary point a supporting hyperplane. This fact gives a set of inequalities for $a_{2}, \ldots, a_{n+1}$ relative to a boundary point $\left(a_{2}^{0}, \ldots, a_{n+1}^{0}\right)$ of $S_{n+1}^{*}$. Among them there are coefficient inequalities for the class $S^{*}$ (Theorem 2) which are quite similar to those the extended general coefficient theorem of J. A. Jenkins gives for the class $S$. They imply that some sections of $S_{n}^{*}$ are convex, i.e. if $a^{0}=\left(a_{2}^{0}, \ldots, a_{\varrho}^{0}\right)$ is a point of $S_{\varrho}^{*}$ and if $W_{n}\left(a_{2}^{0}, \ldots, a_{\varrho}^{0}\right)$ is the set of points $\left(a_{\varrho+1}, \ldots, a_{n}\right)$ in $C^{n-\varrho}$ such that $\left(a_{2}^{0}, \ldots, a_{\varrho}^{0}, a_{\varrho+1}, \ldots, a_{n}\right)$ is in $S_{n}^{*}$, then $W_{n}\left(a_{2}^{0}, \ldots, a_{\varrho}^{0}\right)$ is strictly convex for each point $a^{0}$ in $S_{\varrho}^{*}$ provided that $n \leqq 2 \varrho$, and this bound for $n$ is sharp.

\section{The $n$-th coefficient body}

1.1. In this section we will prove the following

Theorem 1. The n-th coefficient body $S_{n}^{*}$ is homeomorphic to a ball in $C^{n-1}$. For each point $a$ on the boundary of $S_{n}^{*}$ there is only one function in $S^{*}$ which is taken onto a by the mapping $A_{n}$ while $A_{n}^{-1}(a)$ is an infinite set in $S^{*}$ if $a$ is in the interior of $S_{n}^{*} . A_{n}(f)$ is on the boundary of $S_{n}^{*}$ if and only if there are distinct points $\varkappa_{1}, \ldots, \varkappa_{m}$ on the unit circumference $\{|z|=1\}$ and positive numbers $\mu_{1}, \ldots, \mu_{m}$ where $\sum_{j=1}^{m} \mu_{j}=1$ and $1 \leqq m<n$ such that

$$
f(z)=z \prod_{1}^{m}\left(1-x_{j} z\right)^{-2 \mu_{j}} .
$$

Given the boundary point $a$, the numbers $m, \varkappa_{j}$ and $\mu_{j}$ are unique.

$\mathrm{R}$ e m a r k. $f$ takes the unit disc onto a domain which is bounded by $m$ rays $(0,3)$, where $\alpha_{j+1}-\alpha_{j}=2 \pi \mu_{j}, j=1, \ldots, m$. Conversely, any $m$ such rays, $1 \leqq m<n$, up to a suitable homothety $z \rightarrow r z, r>0$, determine via the mapping function a point on the boundary of $S_{n}^{*}$.

Since $f$ belongs to $S^{*}$ if and only if $z f^{\prime}(z) / f(z)$ is in $C$, the differential equation

$$
z f^{\prime}(z)=g(z) f(z)
$$

establishes a homeomorphism between $C$ and $S^{*}$ if $C$ and $S^{*}$ are provided with the topology of uniform convergence on compact subsets of $D$. Equation (1.2) implies the following relations between the coefficients $a_{j}$ and $c_{j}$ in (0.1) and (0.4) respectively: 


$$
\begin{aligned}
a_{2} & =2 c_{1}, \\
2 a_{3} & =2\left(c_{2}+a_{2} c_{1}\right), \\
(n-1) a_{n} & =2\left(c_{n-1}+a_{2} c_{n-2}+\ldots+a_{n-1} c_{1}\right) .
\end{aligned}
$$

For each $n, n=2,3, \ldots$, they define a homeomorphism of $S_{n}^{*}$ onto $C_{n-1}$. Hence, for some basic properties of $S_{n}^{*}$, it suffices to study $C_{n-1}$.

1.2. The following result is due to C. Carathéodory [1] and O. Toeplitz [10] (cf. also [2]).

Th e o r e m A. $C_{n}$ is a convex body in $C^{n}$ containing the origin. To each point $\zeta=\left(c_{1}, \ldots, c_{n}\right)$ in the interior of $C_{n}$ there correspond infinitely many functions in $C$, i.e. $\gamma_{n}^{-1}(\zeta)$ is infinite; but for each point $\zeta$ on the boundary of $C_{n}$ there is only one $g$ in $C$ which is taken onto $\zeta$ by the mapping $\gamma_{n} \cdot \gamma_{n}(g)$ is on the boundary of $C_{n}$ if and only if there are distinct points $x_{1}, \ldots, x_{m}$ on the unit circumference $\{|z|=1\}$ and positive numbers $\mu_{1}, \ldots, \mu_{m}$ such that

$$
g(z)=\sum_{j=1}^{m} \frac{1+x_{j} z}{1-x_{j} z} \mu_{j},
$$

where $1 \leqq m \leqq n$ and $\sum_{j=1}^{m} \mu_{j}=1$. The numbers $m, \varkappa_{j}, \mu_{j}$ are determined uniquely by the boundary point $\zeta$.

Proof of Theorem 1. Theorem A, together with (1.2) and (1.3), immediately implies Theorem 1. Since (1.3) establishes a homeomorphism between the boundaries of $C_{n-1}$ and $S_{n}^{*}$, by integration of (1.2), the functions (1.4) give exactly those functions in $S^{*}$ which are taken onto the boundary of $S_{n}^{*}$ under the mapping $A_{n}$.

1.3. The implication of Theorem 1 for extremal problems within the class $S^{*}$ is immediate. Let $F\left(a_{2}, \ldots, a_{n}\right)$ be a real valued function of the complex variables $a_{2}, \ldots, a_{n}$, which is defined and continuously differentiable with respect to the real variables $x_{j}=\operatorname{Re} a_{j}, y_{j}=\operatorname{Im} a_{j}$, $j=2, \ldots, n$, in some neighborhood $N$ of $S_{n}^{*}$, such that $|\operatorname{grad} F|$ is positive there. Then the function $F$ attains its maximum on $S_{n}^{*}$ only on the boundary. Hence each function $f$ which maximizes $F$ (considered as a functional on $S^{*}$ ) on $S^{*}$ is necessarily of type (1.1). This result was proved by J. A. Hummel by variational methods of Schiffer's type within the class $S^{*}$ (cf. [4]).

Furthermore, let $F$ attain its maximum at a point $\left(a_{2}^{0}, \ldots, a_{n}^{0}\right)$ of the boundary $\partial S_{n}^{*}$. Then, from 


$$
\begin{aligned}
F\left(a_{2}, \ldots, a_{n}\right)= & F\left(a_{2}^{0}, \ldots, a_{n}^{0}\right)+2 \operatorname{Re} \sum_{j=2}^{n} \frac{\partial F}{\partial a_{j}} \Delta a_{j} \\
& +o\left(\max _{j}\left\{\left|\Delta a_{j}\right|\right\}\right), \quad \Delta a_{j}=a_{j}-a_{j}^{0},
\end{aligned}
$$

it follows that

$$
\operatorname{Re}\left\{\sum_{2}^{n} \frac{\partial F}{\partial a_{j}}\left(a_{2}^{0}, \ldots, a_{n}^{0}\right) \Delta a_{j}\right\}+o\left(\max _{j}\left\{\left|\Delta a_{j}\right|\right\}\right) \leqq 0
$$

and this shows that $\left(\partial F / \partial a_{2}, \ldots, \partial F / \partial a_{n}\right)$ is an outer normal vector to $S_{n}^{*}$ at $\left(a_{2}^{0}, \ldots, a_{n}^{0}\right)$. (Cf. also Paragraph 2.1.)

1.4. If $\zeta=\left(c_{1}, \ldots, c_{n}\right)$ is an interior point of $C_{n}$, then $\gamma_{n}^{-1}(\zeta)$ is an infinite set in $C$. It is possible, however, to define in a natural way a subset of $C$ which is homeomorphic to $C_{n}$ under the mapping $\gamma_{n}$. Assume first that $\zeta \neq(0, \ldots, 0)$. Since $C_{n}$ is a convex body containing the origin, there is a unique number $t>1$ such that $t \zeta$ is on the boundary of $C_{n}$. By Theorem A there is a unique set of numbers $\varkappa_{j}$ and $\mu_{j}, j=1, \ldots, m$, $1 \leqq m \leqq n$, such that

$$
g(z)=\sum_{j=1}^{m} \frac{1+\varkappa_{j} z}{1-\varkappa_{j} z} \mu_{j}
$$

corresponds to $t \zeta$, i.e. $\gamma_{n}(g)=t \zeta$. The function $g^{*}=g / t+1-1 / t$, which can be written in the form

$$
g^{*}(z)=1+2 \sum_{j=1}^{m} \frac{\varkappa_{j} z}{1-\varkappa_{j} z} \mu_{j}^{*}, \quad \sum_{1}^{m} \mu_{j}^{*}=\frac{1}{t},
$$

is in $C$ and $\gamma_{n}\left(g^{*}\right)=\zeta$. If $\zeta=(0, \ldots, 0)$ we choose $1 / t=0$, i.e. $\mu$ vanishes and $g^{*}$ is just the constant 1 , which case may be characterized also by setting $m=0$. Thus we proved

Th e o r e m $\mathrm{A}^{\prime}$. To each point $\zeta=\left(c_{1}, \ldots, c_{n}\right)$ of $C_{n}$ there corresponds a unique set of distinct points $\varkappa_{1}, \ldots, \varkappa_{m}$ on the unit circumference and a set of positive numbers $\mu_{1}, \ldots, \mu_{m}$, where $\sum_{j=1}^{m} \leqq 1$ and $0 \leqq m \leqq n$, such that

$$
g(z ; \zeta)=1+2 \sum_{j=1}^{m} \frac{\varkappa_{j} z}{1-\varkappa_{j} z} \mu_{j}
$$

is in $C$ and $\gamma_{n}(g(. ; \zeta))=\zeta$. The set of the numbers $x_{j}$ and $\mu_{j}$ might be empty in which case we set $m=0$. $\zeta$ is on the boundary of $C_{n}$ if and only if $\sum_{1}^{m} \mu_{j}=1$. The correspondence $\zeta \mapsto g(. ; \zeta)$ defines a homeomorphic mapping of $C_{n}$ onto the subset $\{g(. ; \zeta)\}$ of $C$. 
As before, we obtain from this Theorem $\mathrm{A}^{\prime}$, together with (1.3) and (1.2),

$\mathrm{Th}$ e o r e m 1'. Let $M$ denote the set of measures defined by $m$ distinct points $\varkappa_{1}, \ldots, x_{m}$ on the unit circumference with assigned positive numbers $\mu_{1}, \ldots, \mu_{m}$ such that $\sum_{j}^{m} \mu_{j} \leqq 1$ and $0 \leqq m<n$. Then by (1.1) there corresponds to $M$ a subset of functions in $S^{*}$ which is homeomorphic to $S_{n}^{*}$.

\section{Coefficient inequalities}

2.1. Here we derive coefficient inequalities relative to a given boundary point $a^{0}=\left(a_{2}^{0}, \ldots, a_{n}^{0}\right)$ of $S_{n}^{*}$. By $(1.3)$ there corresponds to $a^{0}$ a point $\zeta^{0}=\left(c_{1}^{0}, \ldots, c_{n-1}^{0}\right)$ on the boundary of $C_{n-1}$. Choose a supporting hyperplane at $\zeta^{0}$, with normal direction $\alpha=\left(\alpha_{1}, \ldots, \alpha_{n-1}\right)$. Then, for any point $\zeta=\left(c_{1}, \ldots, c_{n-1}\right)$ of $C_{n-1}$ we have the inequality

$$
\operatorname{Re}<\zeta-\zeta^{0}, \alpha>\leqq 0 \text {. }
$$

Since by (1.3) $c_{j}-c_{j}^{0}$ depends on the coefficients $a_{2}, a_{2}^{0}, \ldots, a_{j+1}, a_{j+1}^{0}$ only, (2.1) represents an inequality involving the boundary point $a^{0}$ and an arbitrary point $a$ of $S_{n}^{*}$. We transform now (2.1) considering that $\zeta-\zeta^{0}$ could be small. Let $g$ and $g_{0}$ be functions in $C$ such that $\gamma_{n-1}(g)=\zeta$ and $\gamma_{n-1}\left(g_{0}\right)=\zeta^{0}:$

$$
g(z)=\sum_{0}^{\infty} 2 c_{j} z^{j}, \quad g_{0}(z)=\sum_{0}^{\infty} 2 c_{j}^{0} z^{j}, \quad 2 c_{0}=1 .
$$

Define

$$
\Phi(z)=\int_{0}^{z}\left(g(t)-g_{0}(t)\right) \frac{d t}{t}=\varphi_{1} z+\ldots+\varphi_{n} z^{n}+\ldots
$$

where $2\left(c_{j}-c_{j}^{0}\right)=j \varphi_{j}, j=1,2, \ldots$. Let the functions $f$ and $f_{0}$ in $S^{*}$ correspond to $g$ and $g_{0}$. Equation (1.2) implies then

$$
f(z)=z \exp \int_{0}^{z}(g(t)-1) \frac{d t}{t}=f_{0}(z) \exp \Phi(z) .
$$

Write now

$$
\begin{gathered}
\alpha(z)=\frac{\alpha_{n-1}}{z^{n-1}}+\ldots+\frac{\alpha_{1}}{z} \\
k(z)=-\frac{\alpha^{\prime}(z)}{f_{0}(z)}=\frac{k_{n}}{z^{n+1}}+\ldots+\frac{k_{2}}{z^{3}}+\ldots
\end{gathered}
$$


and correspondingly $\bar{\alpha}(z)=\overline{\alpha(\bar{z})}, \quad \bar{k}(z)=\overline{k(\bar{z})}$. It follows then

$$
\begin{gathered}
2<\zeta-\zeta^{0}, \alpha>=\frac{1}{2 \pi i} \oint \bar{\alpha}(z)\left(g(z)-g_{0}(z)\right) \frac{d z}{z} \\
=-\frac{1}{2 \pi i} \oint \bar{\alpha}^{\prime}(z) \Phi(z) d z=\frac{1}{2 \pi i} \oint \bar{k}(z) f_{0}(z) \Phi(z) d z
\end{gathered}
$$

and by (2.1)

$$
\operatorname{Re} \frac{1}{2 \pi i} \oint \bar{k}(z) f_{0}(z) \Phi(z) d z \leqq 0
$$

where the integration is taken along a positively oriented circuit around the origin.

2.2. Choose a variation of $f_{0}$ in $S^{*}$, i.e. a mapping $\varepsilon \mapsto f_{\varepsilon}$ of some interval $\left(0, \varepsilon_{0}\right)$ into $S^{*}$ such that

$$
f_{\varepsilon}=f_{0}+\varepsilon f_{1}+o(\varepsilon),
$$

where $f_{1}$ is holomorphic in $D$ with $f_{1}(z)=O\left(z^{2}\right)$, and $o(\varepsilon) / \varepsilon$ converges to zero uniformly on compact subsets of $D$. If

$$
f_{\varepsilon}(z)=\sum_{j=1}^{\infty} a_{j}(\varepsilon) z^{j} \text { and } f_{1}(z)=\sum_{j=2}^{\infty} a_{j}^{\prime} z^{j},
$$

then $a^{\prime}=\left(a_{2}^{\prime}, \ldots, a_{n}^{\prime}\right)$ is a tangent vector to the curve $\varepsilon \mapsto a(\varepsilon)=$ $\left(a_{2}(\varepsilon), \ldots, a_{n}(\varepsilon)\right), \quad 0 \leqq \varepsilon \leqq \varepsilon_{0} \quad$ at $\quad a(0): \quad a(\varepsilon)=a(0)+\varepsilon a^{\prime}+o(\varepsilon)$ for $\varepsilon \rightarrow 0$. Corresponding to (2.8) we have

$$
g_{\varepsilon}=g_{0}+\varepsilon g_{1}+o(\varepsilon) \text { and } \Phi_{\varepsilon}=\varepsilon \Phi_{1}+o(\varepsilon),
$$

where $\Phi_{1}(z)=\int_{0}^{z} g_{1}(\zeta) d \zeta / \zeta$. From (2.2) and (2.3) it then follows that

$$
f_{1}=f_{0} \Phi_{1}
$$

With $g_{\varepsilon}(z)=\sum_{j=0}^{\infty} c_{j}(\varepsilon) z^{j}$ and $g_{1}(z)=\sum_{j=1}^{\infty} c_{j}^{\prime} z^{j}$ we get $\zeta(\varepsilon)=\zeta(0)+$ $\varepsilon \zeta^{\prime}+o(\varepsilon)$ for $\varepsilon \rightarrow 0$, where $\zeta(\varepsilon)=\left(c_{1}(\varepsilon), \ldots, c_{n-1}(\varepsilon)\right)$ and $\zeta^{\prime}=$ $\left(c_{1}^{\prime}, \ldots, c_{n-1}^{\prime}\right)$. If $\alpha=\left(\alpha_{1}, \ldots, \alpha_{n-1}\right)$ is an outer normal vector to $C_{n-1}$ at the boundary point $\zeta(0)$, it follows from $(2.1),(2.6),(2.9)$ and $(2.2)$ that

$$
\operatorname{Re} \frac{1}{2 \pi i} \oint \bar{k}(z) f_{1}(z) d z=\operatorname{Re}\left\{\bar{k}_{2} a_{2}^{\prime}+\ldots+\bar{k}_{n} a_{n}^{\prime}\right\} \leqq 0
$$

for all tangent vectors $a^{\prime}$, i.e. $\left(k_{2}, \ldots, k_{n}\right)$ is an outer normal vector to $S_{n}^{*}$ at $a^{0}$. 
Conversely, let $k$ be an outer normal to $S_{n}^{*}$ at $a(0)$. For any $g \in C$ define $g_{\varepsilon}=g_{0}+\varepsilon\left(g-g_{0}\right), 0 \leqq \varepsilon \leqq 1$. This is a variation of $g_{0}$ in $C$. It infers a variation $f_{\varepsilon}=f_{0}+\varepsilon f_{1}+o(\varepsilon)$ of $f_{0}$ in $S^{*}$, and a curve $\varepsilon \mapsto a(\varepsilon)=a(0)+\varepsilon a^{\prime}+o(\varepsilon)$ in $S_{n}^{*}$. Let now the vector $\alpha=\left(\alpha_{1}, \ldots, \alpha_{n-1}\right)$ be given by (2.5) and (2.4). From (2.6) and (2.10) it follows that $\operatorname{Re} \sum_{j=1}^{n-1} \bar{\alpha}_{j}\left(c_{j}-c_{j}^{0}\right) \leqq 0$ for all points $\zeta=\left(c_{1}, \ldots, c_{n-1}\right)$ of $C_{n-1}$ and this shows that $\alpha=\left(\alpha_{1}, \ldots, \alpha_{n-1}\right)$ is an outer normal vector to $C_{n-1}$. Thus, by (2.5), we proved

Proposition 2.2. There is a one to one correspondence between the outer normal vectors to $C_{n-1}$ and $S_{n}^{*}$ at associated boundary points, which is given by the equations

$$
\begin{aligned}
\alpha_{1} & =k_{n} a_{n-1}+k_{n-1} a_{n-2}+\ldots+k_{3} a_{2}+k_{2} \\
2 \alpha_{2} & =k_{n} a_{n-2}+k_{n-1} a_{n-3}+\ldots+k_{3}
\end{aligned}
$$

$$
\begin{aligned}
& (n-2) \alpha_{n-2}=k_{n} a_{2}+k_{n-1} \\
& (n-1) \alpha_{n-1}=k_{n} .
\end{aligned}
$$

2.3. The preceding considerations suggest to develop (2.3) into powers of $\Phi$ and to write (2.7) in the form

$$
\operatorname{Re}\left\{\frac{1}{2 \pi i} \oint \bar{k}(z)\left(f(z)-f_{0}(z)-\frac{1}{2 !} f_{0}(z) \Phi^{2}(z)-\ldots\right) d z\right\} \leqq 0
$$

Only the powers $\Phi^{2}, \ldots, \Phi^{n-1}$ are relevant for the evaluation of the integral, since $\Phi$ has a zero at the origin. However, the higher is the order of this zero the less powers of $\Phi$ are needed. Observe that by $(2.2)$ we have

$$
\Phi(z)=\varphi_{\varrho} z^{\varrho}+\varphi_{\varrho+1} z^{\varrho+1}+\ldots, \quad 1 \leqq \varrho<n,
$$

or $c_{j}=c_{j}^{0}$ for $j=1, \ldots, \varrho-1$ if and only if $a_{j}=a_{j}^{0}, j=2, \ldots, \varrho$, and that in case of (2.13) we have

$$
\begin{aligned}
\bar{k}(z) f_{0}(z) \Phi^{2}(z) & =\left(\frac{\bar{k}_{n}}{z^{n+1}}+\ldots\right)\left(z+a_{2}^{0} z^{2}+\ldots\right)\left(\varphi_{\varrho}^{2} z^{2 \varrho}+\ldots\right) \\
& =\bar{k}_{n} \varphi_{\varrho}^{2} z^{2 \varrho-n}+\ldots
\end{aligned}
$$

We consider two cases.

$1^{\circ} 2 \varrho \geqq n$. In this case inequality $(2.12)$ reduces to

$$
\operatorname{Re}\left\{\sum_{j=\varrho+1}^{n} \bar{k}_{j}\left(a_{j}-a_{j}^{0}\right)\right\} \leqq 0 .
$$


Equality occurs if and only if it holds in (2.7) also. $k=\left(k_{2}, \ldots, k_{n}\right)$ is an outer normal vector to $S_{n}^{*}$ at the point $a^{0}=\left(a_{2}^{0}, \ldots, a_{n}^{0}\right)$. To $a^{0}$ and $k$ there corresponds the outer normal vector $\alpha=\left(\alpha_{1}, \ldots, \alpha_{n-1}\right)$ to $C_{n-1}$ at $\zeta^{0}=\left(c_{1}^{0}, \ldots, c_{n-1}^{0}\right)$. From (2.6) it follows that equality holds in (2.7) if and only if

$$
\operatorname{Re}\left\langle\zeta-\zeta^{0}, \alpha\right\rangle=\operatorname{Re} \sum_{j=1}^{n-1}\left(c_{j}-c_{j}^{0}\right) \bar{\alpha}_{j}=0,
$$

i.e. the point $\zeta=\left(c_{1}, \ldots, c_{n-1}\right)$ lies on the supporting hyperplane through $\zeta^{0}$ with normal direction $\alpha$. Since, by assumption, $c_{j}=c_{j}^{0}$ for $j=$ $1, \ldots, \varrho-1$ and $2 \varrho \geqq n$ it follows from Lemma 3.5 (in Paragraph 3.5, with $\varrho-1$ and $n-1$ instead of $\varrho$ and $n$ respectively) that this occurs only if $\zeta=\zeta^{0}$, hence only if $a=a^{0}$ or $f=f_{0}$.

$2^{\circ} n=2 \varrho+1$. In this case the residue of $\bar{k}(z) f_{0}(z) \Phi^{2}(z)$ at the origin is $\bar{k}_{n} \varphi_{\varrho}^{2}$ and from (2.3) it follows $\varphi_{\varrho}=a_{\varrho+1}-a_{\varrho+1}^{0}$. Hence (2.12) implies

$$
\operatorname{Re}\left\{\sum_{j=\varrho+1}^{n} \bar{k}_{j}\left(a_{j}-a_{j}^{0}\right)-\frac{\bar{k}_{n}}{2}\left(a_{\varrho+1}-a_{\varrho+1}^{0}\right)^{2}\right\} \leqq 0 .
$$

If $a_{\varrho+1}=a_{\varrho+1}^{0}$ equality occurs only if $a=a^{0}$, because then we are in the preceding case, i.e. $n<2(\varrho+1)$. Thus we proved

Th e o r e m 2. Let $a^{0}=\left(a_{2}^{0}, \ldots, a_{n}^{0}\right)$ be a boundary point of the coefficient body $S_{n}^{*}$, let $k=\left(k_{2}, \ldots, k_{n}\right)$ be an outer normal vector to $S_{n}^{*}$ at $a^{0}$ and let the integer $\varrho$ satisfy the condition $\varrho<n \leqq 2 \varrho+1$. If $\varepsilon=0$ for $n \leqq 2 \varrho$ and $\varepsilon=1$ for $n=2 \varrho+1$, then the inequality

$$
\operatorname{Re}\left\{\sum_{j=\varrho+1}^{n} \bar{k}_{j}\left(a_{j}-a_{j}^{0}\right)-\varepsilon \frac{\bar{k}_{n}}{2}\left(a_{\varrho+1}-a_{\varrho+1}^{0}\right)^{2}\right\} \leqq 0
$$

holds for all points a of $S_{n}^{*}$ such that $a_{j}=a_{j}^{0}, j=2, \ldots, \varrho$. In the case that $n \leqq 2 \varrho$ or that $n=2 \varrho+1$ and $a_{\varrho+1}=a_{\varrho+1}^{0}$ equality occurs in (2.16) if and only if $f=f_{0}$.

$\mathrm{R}$ e $\mathrm{m}$ a r k. Theorem 2 gives a coefficient inequality which is quite similar to the one J. A. Jenkins has given in his general coefficient Theorem [5] for the particular case of the normalized schlicht functions in the unit dise.

2.4. In the case $n \leqq 2 \varrho$ Theorem 2 has an interesting corollary. Choose in $S_{\varrho}^{*}$ a fixed point $\left(a_{2}^{0}, \ldots, a_{\varrho}^{0}\right), 1<\varrho<n$. Denote by $W_{n}\left(a_{2}^{0}, \ldots, a_{\varrho}^{0}\right)$ the set of all points $\left(a_{\varrho+1}, \ldots, a_{n}\right)$ in $C^{n-\varrho}$ such that $\left(a_{2}^{0}, \ldots, a_{\varrho}^{0}, a_{\varrho+1}, \ldots, a_{n}\right)$ is in $S_{n}^{*}$. Let $\left(a_{\varrho+1}^{0}, \ldots, a_{n}^{0}\right)=A^{0}$ be on the boundary of $W_{n}$. Then $\left(a_{2}^{0}, \ldots, a_{\varrho}^{0}, a_{\varrho+1}^{0}, \ldots, a_{n}^{0}\right)=a^{0}$ is a boundary point of $S_{n}^{*}$. Choose there an outer normal vector $k=\left(k_{2}, \ldots, k_{n}\right)$. By Theorem 2 we have $(2.15)$ for all points $\left(a_{\varrho+1}, \ldots, a_{n}\right)$ in $W_{n}\left(a_{2}^{0}, \ldots, a_{\varrho}^{0}\right)$. 
This shows that at each boundary point $W_{n}$ has a supporting hyperplane, hence $W_{n}$ is convex. It is even strictly convex, i.e. each supporting hyperplane to $W_{n}$ contains only one point of $W_{n}$ because equality occurs in (2.15) only if $a_{j}=a_{j}^{0}, j=\varrho+1, \ldots, n$.

Now we show that $W_{n}\left(a_{2}, \ldots, a_{\varrho}\right)$ is no more convex for arbitrary points $\left(a_{2}, \ldots, a_{\varrho}\right)$ in $S_{\varrho}^{*}$, if $n>2 \varrho$. More precisely, we show that $W_{2 \varrho+1}(0, \ldots, 0)$ is not convex. For this purpose we consider the two functions

$$
\begin{aligned}
& f_{1}(z)=\left(k\left(z^{\varrho}\right)\right)^{1 / \varrho}=z+\frac{2}{\varrho} z^{\varrho+1}+\frac{\varrho+2}{\varrho^{2}} z^{2 \varrho+1}+\ldots \text { and } \\
& f_{2}(z)=\varepsilon^{-1} f_{1}(\varepsilon z)=z-\frac{2}{\varrho} z^{\varrho+1}+\frac{\varrho+2}{\varrho^{2}} z^{2 \varrho+1}+\ldots
\end{aligned}
$$

in $S^{*}$, where $\varepsilon^{\varrho}=-1$ and $k$ is the Koebe function $k(z)=z /(1-z)^{2}$. They show that $W_{2 \varrho+1}(0, \ldots, 0)$ contains the points

$$
\left(2 / \varrho, 0, \ldots, 0,(\varrho+2) / \varrho^{2}\right) \text { and }\left(-2 / \varrho, 0, \ldots, 0,(\varrho+2) / \varrho^{2}\right) \text {. }
$$

But the midpoint $\left(0, \ldots, 0,(\varrho+2) / \varrho^{2}\right)$ of them does not belong to $W_{2 \varrho+1}(0, \ldots, 0)$, because for any schlicht function, hence for any starlike function $f(z)=z+a_{2 \varrho+1} z^{2 \varrho+1}+\ldots$ we have $\left|a_{2 \varrho+1}\right| \leqq 1 / \varrho$ by a result of Prawitz ([8]) and because $1 / \varrho<(\varrho+2) / \varrho^{2}$. Thus we proved

$\mathrm{T}$ h e o r e m 3. Associate to a point $\left(a_{2}^{0}, \ldots, a_{\varrho}^{0}\right)$ in $S_{\varrho}^{*}$ and an integer $n>\varrho$ the set $W_{n}\left(a_{2}^{0}, \ldots, a_{\varrho}^{0}\right)$ of those points $\left(a_{\varrho+1}, \ldots, a_{n}\right)$ in $C^{n-\varrho}$ for which $\left(a_{2}^{0}, \ldots, a_{\varrho}^{0}, a_{\varrho+1}, \ldots, a_{n}\right)$ is in $S_{n}^{*}$. Then $W_{n}\left(a_{2}^{0}, \ldots, a_{\varrho}^{0}\right)$ is a strictly convex body if $n \leqq 2 \varrho$. However, $W_{2 \varrho+1}(0, \ldots, 0)$ is no longer convex.

A similar theorem holds for the coefficient bodies of the class $S$ (cf. [7]).

Let consider the particular case that $\varrho=n-1$. If $\left(a_{2}^{0}, \ldots, a_{n-1}^{0}\right)$ is on the boundary of $S_{n-1}^{*}$, then obviously $W_{n}\left(a_{2}^{0}, \ldots, a_{n-1}^{0}\right)$, the range of $a_{n}$, is a point. Thus we may assume that $\left(a_{2}^{0}, \ldots, a_{n-1}^{0}\right)$ is in the interior of $S_{n-1}^{*}$. The corresponding point $\left(c_{1}^{0}, \ldots, c_{n-2}^{0}\right)$ is in the interior of $C_{n-2}$. As was remarked by Carathéodory ([1]), the range of $C_{n}$ is a disc. Hence, by (1.3), it follows: For a given point $\left(a_{2}^{0}, \ldots, a_{n-1}^{0}\right)$ in $S_{n-1}^{*}$ the range of $a_{n}$ is either a disc or a point. Based on a different method this result was given by J.A. Hummel in [3].

\section{Proof of Theorem A}

3.1. $C_{n}$ is a compact and convex set in $C^{n}$, since $C$ is convex and compact (in the topology of uniform convergence on compact subsets of $D$ ), and $\gamma_{n}$ is continuous and linear. 
3.2. Let $\zeta$ be an interior point of $C_{n}$. There is a $\lambda, \lambda>1$, such that $\lambda \zeta=\left(\lambda c_{1}, \ldots, \lambda c_{n}\right)$ is still in $C_{n}$. Choose in $C$ a function $g$ such that $\gamma_{n}(g)=\lambda \zeta$. Then $g_{1}=\lambda^{-1} g+(1-1 / \lambda)$ is in $C$ and satisfies $\operatorname{Re} g_{1}(z)>1-1 / \lambda$. Hence, $g_{1}+h$ is in $C$ and $\gamma_{n}\left(g_{0}+h\right)$ equals to $\zeta$ for each function $h(z)=b_{n+1} z^{n+1}+\ldots$ which is holomorphic in $D$, such that $\sup _{z \in D}|h(z)| \leqq 1-1 / \lambda$. This proves that $\gamma_{n}^{-1}(\zeta)$ is an infinite set in $C$.

3.3. Let $P$ denote the set of probability measures supported by the unit circumference $\{|z|=1\}$. According to a result of Herglotz $g$ belongs to the class $C$ if and only if

$$
g(z)=\int_{0}^{2 \pi} \frac{1+e^{i \theta} z}{1-e^{i \theta} z} d \mu_{\theta}, \quad \mu \in P
$$

or equivalently if and only if the coefficients $c_{n}$ of $g($ in $(0,4))$ are the trigonometric moments of a probability measure, i.e.

$$
c_{n}=\int_{0}^{2 \pi} e^{i n \theta} d \mu_{\theta}, \quad \mu \in P, \quad n=0,1,2, \ldots
$$

In the sequel we represent points in $\mathbb{R}^{2 n}$ in the form $\zeta=\left(\zeta_{1}, \ldots, \zeta_{n}\right)$, $\zeta_{j} \in C$, as points in $C^{n}$, and consequently, we write the standard scalarproduct in $\mathbb{R}^{2 n}$ as $\operatorname{Re}\left\langle\zeta, \zeta^{1}\right\rangle=\operatorname{Re} \sum_{j=1}^{n} \bar{\zeta}_{j} \zeta_{j}^{\prime}$.

Hence, the norm of $\zeta$ is given by $|\zeta|=(\langle\zeta, \zeta\rangle)^{1 / 2}$.

Defining

$$
\zeta(\mu)=\int_{0}^{2 \pi}\left(e^{i \theta}, \ldots, e^{i n \theta}\right) d \mu_{\theta}
$$

for any real measure (supported by the unit circumference) we have

$$
C_{n}=\{\zeta(\mu) \mid \mu \in P\},
$$

i.e. $C_{n}$ is the convex hull of the curve

$$
\Gamma: \theta \mapsto\left(e^{i \theta}, \ldots, e^{i n \theta}\right), \quad 0 \leqq \theta \leqq 2 \pi .
$$

Let $\alpha$ be a unit vector:

$$
\alpha=\left(\alpha_{1}, \ldots, \alpha_{n}\right), \quad|\alpha|=1 .
$$

Define

$$
h(\alpha)=\max _{\theta} \operatorname{Re}\left\{\bar{\alpha}_{1} e^{i \theta}+\ldots+\bar{\alpha}_{n} e^{i n \theta}\right\}
$$

and 


$$
T\left(e^{i \theta}, \alpha\right)=h(\alpha)-\operatorname{Re}\left\{\sum_{j=1}^{n} \bar{\alpha}_{j} e^{i j \theta}\right\} .
$$

Obviously $T\left(e^{i \theta}, \alpha\right) \geqq 0$ for all $\theta$ and $\alpha$. Hence, by a lemma of Fejer and Riesz, there is a polynomial $p(z)=\xi_{0}+\xi_{1} z+\ldots+\xi_{n} z^{n}$ such that

$$
T\left(e^{i \theta}, \alpha\right)=\left|p\left(e^{i \theta}\right)\right|^{2} .
$$

Let now $\zeta_{0}=\zeta\left(\mu_{0}\right), \mu_{0} \in P$, be a point on the boundary of $C_{n}$. Since $C_{n}$ is convex there is a supporting hyperplane

$\operatorname{Re}\left\langle\zeta-\zeta_{0}, \alpha>=0,|\alpha|=1\right.$, i.e. $\max _{\zeta \in C_{n}} \operatorname{Re}\left\langle\zeta, \alpha>=\operatorname{Re}\left\langle\zeta_{0}, \alpha\right\rangle\right.$.

Since $C_{n}$ is the convex hull of $\Gamma$ we have also

$$
\max _{\theta} \operatorname{Re} \sum_{j=1}^{n} \bar{\alpha}_{j} e^{i j \theta}=\operatorname{Re}<\zeta_{0}, \alpha>
$$

or by $(3.3) h(\alpha)-\operatorname{Re}\left\langle\zeta_{0}, \alpha>=0\right.$. With the notations (3.4) and (3.5) and with (3.1) it follows

$$
\int_{0}^{2 \pi} T\left(e^{i \theta}, \alpha\right) d \mu_{0}=\int_{0}^{2 \pi}\left|p\left(e^{i \theta}\right)\right|^{2} d \mu_{0}=0
$$

and this shows that a measure $\mu_{0}, \mu_{0} \in P$, such that $\zeta\left(\mu_{0}\right)$ is on the boundary of $C_{n}$, is a measure supported by at most $n$ points on the unit circumference.

Conversely, for an integer $m, 1 \leqq m \leqq n$, choose $m$ distinct points $x_{j}$ on the unit circumference and positives numbers $\mu_{j}, j=1, \ldots, m$ such that $\sum \mu_{j}=1$. Let the pairs $\left\{\left(\varkappa_{j}, \mu_{j}\right)\right\}$ define the measure $\mu_{0}$. Then $\zeta\left(\mu_{0}\right)$ is on the boundary of $C_{n}$. In fact, setting $p(z)=\Pi_{1}^{m}\left(z-x_{j}\right)$ we have

$$
\int_{0}^{2 \pi}\left|p\left(e^{i \theta}\right)\right|^{2} d \mu_{0}=\sum_{1}^{m} p\left(\varkappa_{j}\right) \mu_{j}=0 .
$$

But

$$
\left|p\left(e^{i \theta}\right)\right|^{2}=\alpha_{0}-\operatorname{Re} \sum_{j=1}^{n} \bar{\alpha}_{j} e^{i j \theta}
$$

for suitably chosen numbers $\alpha_{j}$. As a positive factor is not relevant we can assume that $\alpha=\left(\alpha_{1}, \ldots, \alpha_{n}\right)$ is a unit vector and this implies $\alpha_{0}=h(\alpha)$. Equivalently to (3.6) we have then 
$\int_{0}^{2 \pi} T\left(e^{i \theta}, \alpha\right) d \mu_{0}=0$, and $\int_{0}^{2 \pi} T\left(e^{i \theta}, \alpha\right) d \mu \geqq 0$ for all $\mu \in P$, i.e.

$$
\max _{\zeta \in C_{n}} \operatorname{Re}\left\langle\zeta, \alpha>=\operatorname{Re}<\zeta\left(\mu_{0}\right), \alpha>\right.
$$

This shows that $\zeta\left(\mu_{0}\right)$ is on the boundary of $C_{n}$ ending the proof that $\zeta=\gamma_{n}(g)$ belongs to the boundary of $C_{n}$ if and only if $g$ is given by (1.4).

3.4. It will be shown now that for a point $\zeta^{0}=\left(c_{1}, \ldots, c_{n}\right)$ on the boundary of $C_{n}$ there is only one measure $\mu$ in $P$ such that $\zeta(\mu)=\zeta^{0}$, and then this implies that there is a unique $g$ in $C$ satisfying $\gamma_{n}(g)=\zeta_{0}$.

Let $\mu$ be a measure in $P$ such that $\zeta(\mu)=\zeta^{0} \cdot \mu$ is carried by some points $\varkappa_{1}, \ldots, \varkappa_{m}, 1 \leqq m \leqq n$; hence,

$$
\zeta^{0}=\mu_{1} \zeta^{1}+\ldots+\mu_{m} \zeta^{m}, \quad \mu_{j}>0, \quad \sum_{1}^{m} \mu_{j}=1,
$$

where

$$
\zeta^{j}=\left(x_{j}, x_{j}^{2}, \ldots, x_{j}^{n}\right), \quad j=1, \ldots, m .
$$

These vectors $\zeta^{j}$ are linearly independent (because the points $\varkappa_{j}$ are distinct). Their convex hull $\operatorname{coh}\left\{\zeta^{1}, \ldots, \zeta^{m}\right\}$ lies on the $m-1$ dimensional hyperplane

$$
\zeta=\sum_{j=1}^{m} \lambda_{j} \zeta^{j}, \quad \sum_{1}^{m} \lambda_{j}=1
$$

and contains $\zeta^{0}$ in its interior, since all the weights $\mu_{j}$ are positive.

Let now $\mu^{\prime}$ be another measure in $P$ such that $\zeta\left(\mu^{\prime}\right)=\zeta(\mu)=\zeta^{0}$. $\mu^{\prime}$ is carried by some points $\varkappa_{1 j}, j=1, \ldots, m_{1}, 1 \leqq m_{1} \leqq n$ such that

$$
\zeta^{0}=\sum_{j=1}^{m_{1}} \zeta_{1}^{j} \mu_{j}^{\prime}, \quad \mu_{j}^{\prime}>0, \quad \sum_{1}^{m_{1}} \mu_{j}^{\prime}=1,
$$

where

$$
\zeta_{1}^{j}=\left(x_{1 j}, x_{1 j}^{2}, \ldots, x_{1 j}^{n}\right), \quad j=1, \ldots, m_{1} .
$$

Since $\zeta^{0}$ is in the interior of either convex hull, say of $\operatorname{coh}\left\{\zeta^{\prime}, \ldots, \zeta^{m}\right\}$ and of $\operatorname{coh}\left\{\zeta_{1}^{1}, \ldots, \zeta_{1}^{m_{1}}\right\}$, these convex hulls lie on the same hyperplane of dimension $m-1=m_{1}-1$. Choose a $\varkappa_{1 \varrho}, \varrho=1, \ldots, m_{1}$, and write $x_{1 e}=x$. From $\zeta_{1}^{0}=\sum_{j=1}^{m} \lambda_{j} \zeta^{j}, \sum_{1}^{m} \lambda_{j}=1$, according to (3.8) it follows that the vectors $\zeta_{1}^{0}-\zeta^{1}, \zeta^{2}-\zeta^{1}, \ldots, \zeta^{m}-\zeta^{1}$ are linearly dependent, hence 


$$
\left|\begin{array}{c}
x-x_{1}, x_{2}-x_{1}, \ldots, x_{m}-x_{1} \\
x^{2}-x_{1}^{2}, x_{2}^{2}-x_{1}^{2}, \ldots, x_{m}^{2}-x_{1}^{2} \\
\ldots \\
x^{m}-x_{1}^{m}, x_{2}^{m}-x_{1}^{m}, \ldots, x_{m}^{m}-x_{1}^{m}
\end{array}\right|=0 .
$$

Consider this determinant as a polynomial in $\varkappa$. It is of degree $m$ and does not vanish identically; its roots are $x_{1}, \ldots, x_{m}$; hence, $x_{1 \varrho}=x$ equals to one of these roots and this implies that the two sets $\left\{x_{1}, \ldots, x_{m}\right\}$ and $\left\{x_{11}, \ldots, x_{1 m_{1}}\right\}$ are identical, i.e. the two measures $\mu$ and $\mu^{\prime}$ have the same support $\left\{x_{1}, \ldots, x_{m}\right\}$. Their values $\mu_{j}$ carried by the points $x_{j}$ have to satisfy the linear system

$$
\sum_{j=1}^{m} x_{j}^{\lambda} \mu_{j}=c_{\lambda}, \quad \lambda=1, \ldots, n .
$$

Since the matrix $\left(x_{j}^{\lambda}\right)_{j=1, \ldots, m}^{\lambda=1, \ldots, n}$ has rank $m$, the point $\zeta^{0}=\left(c_{1}, \ldots, c_{n}\right)$ uniquely determines the weights $\mu_{j}$ and this implies $\mu^{1}=\mu$. We conclude that for a boundary point $\zeta^{0}$ of $C_{n}$ there is a unique function $g$ in $C$ such that $\gamma_{n}(g)=\zeta^{0}$, and this completes the proof of Theorem A.

3.5. The lemma we used in Paragraph 2.3 easily follows by a similar argument as used just ahead. Let a supporting hyperplane to $C_{n}$, with normal direction $\alpha$, be given. If the polynomial $h(\alpha)-\operatorname{Re} \sum_{j=1}^{n} \bar{\alpha}_{j} z^{j}$ has the zeros $x_{1}, \ldots, x_{m}$ on the unit circumference, then the intersection of $C_{n}$ with the given supporting hyperplane is the convex hull of the points $\zeta^{j}, j=1, \ldots, m$, where the $\zeta^{j}$ are given by (3.7). Furthermore, let the coefficients $c_{1}, \ldots, c_{\varrho}$ be given. With $c_{-k}=\bar{c}_{k}, k=1, \ldots, \varrho$ and $c_{9}=1$ they have to satisfy the equations

$$
\sum_{j=1}^{m} x_{j}^{k} \mu_{j}=c_{k}, \quad k=0, \pm 1, \ldots, \pm \varrho
$$

because the $\mu_{j}$ are real. The matrix $\left(x_{j}^{k}\right)_{k=-\varrho, \ldots, \varrho}^{j=1, \ldots, m}$ has rank $m$ if $2 \varrho+$ $1 \geqq m$. This shows that the coefficients $c_{1}, \ldots, c_{Q}$ uniquely determine the point $\left(c_{1}, \ldots, c_{n}\right)$ on the supporting hyperplane with the given normal direction $\alpha$. Thus we proved

$\mathrm{L}$ e $\mathrm{m} \mathrm{m}$ a 3.5 . Let a given supporting hyperplane to $C_{n}$ touch the curve

$$
\Gamma: \theta \mapsto\left(e^{i \theta}, e^{2 i \theta}, \ldots, e^{i n \theta}\right), \quad 0 \leqq \theta \leqq 2 \pi,
$$

in $m$ distinct points, $1 \leqq m \leqq n$. A point $\left(c_{1}, \ldots, c_{n}\right)$ of $C_{n}$ on this hyperplane is then uniquely determined by its coordinates $c_{1}, \ldots, c_{Q}$, if $2 \varrho+1 \geqq m$. 


\section{References}

[1] CARATHÉOdORY, C.: Über den Variabilitätsbereich der Fourier'schen Konstanten von positiven harmonischen Funktionen. - Rend. Circ. Mat. Palermo 32, 1911, 193-217.

[2] Grenander, U., and G. SzEGö: Toeplitz forms and their applications. California Monographs in Mathematical Sciences, University of California Press, Berkeley - Los Angeles, 1958.

[3] Hummer, J. A.: The coefficient regions of starlike functions. - Pacific J. Math. 7, 1957, 1381-1389.

[4] -》- A variational method for starlike functions. - Proc. Amer. Math. Soc. $9,1958,82-87$.

[5] Jenkins, J. A.: An extension of the general coefficient theorem. - Trans. Amer. Math. Soc. 95, 1960, 387-407.

[6] Nevanlinna, R.: Über die konforme Abbildung von Sterngebieten. - Översikt av Finska vetenskaps-societetens förhandlingar 63. A. 6, Helsingfors, $1920-1921,1-21$.

[7] PFLUGER, A.: О выпуклости некоторых сечений $n$-тел козффициентов однолистных функций. - Некоторые проблемы математики и механики, К семидесятилетию академика М. А. Лаврентъева, Издателъство "Наука", Ленинград, 1970, 233-241. Translation: On the convexity of some sections of the $n$th coefficient body for schlicht functions. - Some problems of mathematics and mechanics, On the occasion of the seventieth birthday of Academician M. A. Lavrent'ev, Amer. Math. Soc. Transl. (2) 104, 1976, 215-222.

[8] PrawiTz, H.: Über Mittelwerte analytischer Funktionen. - Ark. Mat. Astronom. Fys. 20. A. 6, 1927-1928, 1-12.

[9] SCHAEFFER, A. C., and D. C. SPENCER: Coefficient regions for schlicht functions. - American Mathematical Society Colloquium Publications 35, New York, 1950.

[10] TOEPliTz, O.: Über die Fourier'sche Entwickelung positiver Funktionen. Rend. Circ. Mat. Palermo 32, 1911, 191-192.

Eidgenössische Technische Hochschule

Mathematisches Institut

CH 8001 Zürich

Schweiz

Received 27 October 1975 